\title{
Computational Validation of New Reynolds Stress Closure for Nonequilibrium Effects in Turbulent Flows
}

\author{
Peter E. Hamlington and Werner J.A. Dahm \\ Laboratory for Turbulence and Combustion (LTC) \\ Department of Aerospace Engineering, The University of Michigan \\ Ann Arbor, MI, 48109-2140, USA
}

\begin{abstract}
The computational formulation of a new nonequilibrium Reynolds stress closure is presented along with preliminary validation results for both homogeneous and inhomogeneous turbulent flow problems of practical engineering importance. The new nonequilibrium closure, which has been rigorously derived elsewhere ${ }^{1}$ replaces the classical Boussinesq hypothesis appearing in many current two-equation turbulence models with a comparably simple representation for the Reynolds stresses, thereby allowing straightforward implementation in existing computational frameworks. The new nonequilibrium closure has been extended to include a rigorously derived realizable eddy viscosity, and theoretical details of the closure are evaluated through fundamental tests of periodically and impulsively sheared homogeneous turbulence. The full computational formulation of the nonequilibrium closure is outlined for both $k-\epsilon$ and $k-\omega$ model frameworks. Finally, preliminary inhomogeneous flow results are presented using the $k-\omega$ framework for turbulent flow over a flat-plate and the interaction of an impinging oblique shock wave with a turbulent boundary layer.
\end{abstract}

\section{Introduction}

Due to the substantial computational resources required for direct numerical simulations (DNS) and true large eddy simulations (LES) of practical engineering turbulent flow problems, simulation approaches based on the Reynolds-averaged Navier-Stokes (RANS) equations remain the "state of the practice" for these types of flows. The single-point RANS equations are obtained by averaging the continuity and momentum equations, yielding

$$
\begin{aligned}
& \frac{\partial \rho}{\partial t}+\frac{\partial\left(\rho \bar{u}_{i}\right)}{\partial x_{i}}=0, \\
& \rho \frac{D \bar{u}_{i}}{D t}=-\frac{\partial \bar{p}}{\partial x_{i}}+\frac{\partial}{\partial x_{j}}\left[2 \mu \bar{S}_{i j}-\frac{2}{3} \mu \bar{S}_{n n} \delta_{i j}-\rho \overline{u_{i}^{\prime} u_{j}^{\prime}}\right],
\end{aligned}
$$

where $D / D t$ denotes the mean flow material derivative and $\bar{S}_{i j}$ is the symmetric mean strain rate tensor, defined as

$$
\bar{S}_{i j} \equiv \frac{1}{2}\left(\frac{\partial \bar{u}_{i}}{\partial x_{j}}+\frac{\partial \bar{u}_{j}}{\partial x_{i}}\right)
$$

Solving (1)-(3) requires a physically-accurate closure representation for the Reynolds stress tensor $\overline{u_{i}^{\prime} u_{j}^{\prime}}$ appearing in (2), where primes denote fluctuations relative to the average. This stress tensor can be written in terms of its isotropic form $\frac{2}{3} k \delta_{i j}$ and the deviations from isotropy as

$$
\overline{u_{i}^{\prime} u_{j}^{\prime}}=\frac{2}{3} k \delta_{i j}-\left(\overline{u_{i}^{\prime} u_{j}^{\prime}}\right)_{d e v}
$$

where $k \equiv \frac{1}{2} \overline{u_{i}^{\prime} u_{i}^{\prime}}$ is the turbulence kinetic energy. The anisotropic part $\left(\overline{u_{i}^{\prime} u_{j}^{\prime}}\right)_{d e v}$ can then be equivalently expressed in terms of the Reynolds stress anisotropy tensor $a_{i j}$, defined as

$$
a_{i j} \equiv-\frac{\left(\overline{u_{i}^{\prime} u_{j}^{\prime}}\right)_{d e v}}{k}
$$


Within this framework, a suitable model for the Reynolds stress anisotropy in (5) is required to achieve closure of (1)-(4). Second-order models ${ }^{2,3}$ based on the full Reynolds stress transport equation capture much of the relevant physics governing the evolution of the Reynolds stress anisotropy. However, such models have not gained widespread acceptance due to the significant computational load required for the solution of the six coupled partial differential equations involved in such approaches. Issues of numerical stability and ambiguity surrounding the modeling of higher order terms have also contributed to the general resistance to Reynolds stress transport modeling.

The most widely used turbulence models are formulated in a framework that introduces the required closure directly at the level of the second-order single-point moments $\overline{u_{i}^{\prime} u_{j}^{\prime}}$. These are typically based on the classical equilibrium Boussinesq hypothesis, first introduced in 1877, that assumes the deviatoric stresses in (4) to be directly proportional to the mean strain rate tensor $\bar{S}_{i j}$ in (3). The corresponding anisotropy tensor in (5) is then

$$
a_{i j}=-2 \frac{\nu_{T}}{k} \bar{S}_{i j}
$$

where $\nu_{T}$ is an eddy viscosity that must itself be modeled, often with a two-equation formulation, to complete the closure. The vast majority of such two-equation turbulence models differ primarily in how they choose to represent $\nu_{T}$, but fundamentally are based on the equilibrium assumption in (6) that the anisotropy is proportional to the local instantaneous mean strain rate tensor $\bar{S}_{i j}$.

As the computational demands placed upon such two-equation equilibrium RANS models have increased however, model accuracy and sophistication has proceeded at a somewhat slower pace. In particular, there is an increasing need to simulate complex inhomogeneous nonequilibrium flows, where the mean strain rate tensor may be temporally or spatially rapidly varying. Most existing two-equation model frameworks are incapable of accurately predicting nonequilibrium turbulence effects, and this has largely prevented the use of RANS simulations as a viable engineering design tool in certain flow problems. For example, the highly nonequilibrium interaction between turbulence and a shock wave is of substantial importance in the design of next-generation mixed-compression inlets for propulsion systems, yet the interaction is known to be poorly predicted by most existing two-equation RANS models. ${ }^{4}$

The failure of the Boussinesq closure in (6) in nonequilibrium flows is due to the fact that (6) is fundamentally an equilibrium expression for the anisotropy that assumes the anisotropy to depend only on the local instantaneous value of the mean strain rate tensor $\bar{S}_{i j}$. However, such a representation fares poorly when nonequilibrium effects are significant, namely when temporal or spatial variations in flow properties create large Lagrangian time variations in the strain rate. The underlying dynamics of the anisotropy tensor then cannot remain in equilibrium with the rapidly changing mean strain rate tensor, and the direct proportionality in (6) becomes inaccurate.

By contrast, here we present the first computational results obtained using a new nonequilibrium Reynolds stress closure for a range of complex nonequilibrium flow problems. This new closure has been rigorously derived in previous work, ${ }^{1}$ and replaces the equilibrium Boussinesq hypothesis appearing in most current twoequation models with an alternative closure that is comparably simple, and thus can be readily implemented in existing computational frameworks for solving (1)-(4). In the following, the computational formulation of the new nonequilibrium closure approach, which has now been extended to include a rigorously derived realizable eddy viscosity, is presented in full detail for both $k-\epsilon$ and $k-\omega$ model frameworks. The new closure has been implemented in CFL3D, a Navier-Stokes CFD code developed by NASA, ${ }^{5}$ within the standard $k-\omega$ two-equation model framework. ${ }^{6}$ Fundamental tests in periodically and impulsively sheared homogeneous turbulence are used to validate certain aspects of the nonequilibrium closure, and preliminary results obtained using the full computational formulation are presented for turbulent flow over a flat-plate and the interaction of an impinging oblique shock wave with a turbulent boundary layer.

\section{Nonequilibrium Anisotropy Closure}

In the following Section we summarize the derivation of a new anisotropy closure that seeks to include the principal nonequilibrium dynamics of $a_{i j}$, but that can be readily implemented within existing two-equation computational frameworks such as those found in standard codes for solving the RANS equations. This is done by formulating a replacement for the local instantaneous mean strain rate $\bar{S}_{i j}$ that appears in the

equilibrium closure in (6) with a nonequilibrium effective strain rate tensor $\widetilde{S}_{i j}$ that depends on the straining history of the flow. The full derivation of the nonequilibrium closure and the effective strain rate has been 
presented in detail elsewhere, ${ }^{1}$ and in the following we summarize the key steps leading to the time-local computational formulation of the nonequilibrium closure. The accuracy of the time-local formulation is evaluated using periodically-sheared homogeneous turbulence, for which DNS validation data are available from Yu and Girimaji. ${ }^{7}$

\section{II.A. Modeled Anisotropy Transport Equation}

The new nonequilibrium closure and effective strain rate tensor are rigorously derived from the exact transport equation for the anisotropy tensor $a_{i j}$, which is given in general form as $\mathrm{s}^{2,3,8}$

$$
\frac{D a_{i j}}{D t}=-\left(\frac{P}{\epsilon}-1\right) \frac{\epsilon}{k} a_{i j}+\frac{1}{k}\left[P_{i j}-\frac{2}{3} P \delta_{i j}\right]+\frac{1}{k} \Pi_{i j}+\frac{1}{k}\left[D_{i j}-\left(a_{i j}+\frac{2}{3} \delta_{i j}\right) D\right],
$$

where $D / D t$ is the mean-flow Lagrangian derivative, $\epsilon$ is the dissipation rate of the turbulence kinetic energy, $P_{i j}$ is the anisotropy production tensor, $P \equiv P_{l l} / 2$ is the scalar kinetic energy production rate, $\Pi_{i j}$ is the pressure-strain rate correlation tensor, and $D_{i j}$ is the combined viscous and turbulent transport tensor, where $D \equiv D_{l l} / 2$. Using the standard representation for the pressure-strain rate correlation that is linear in $a_{i j}$, namely ${ }^{2,3}$

$$
\Pi_{i j}=-C_{1} \epsilon a_{i j}+C_{2} k \bar{S}_{i j}+C_{3} k\left(a_{i l} \bar{S}_{l j}+\bar{S}_{i l} a_{l j}-\frac{2}{3} a_{n l} \bar{S}_{n l} \delta_{i j}\right)-C_{4} k\left(a_{i l} \bar{W}_{l j}-\bar{W}_{i l} a_{l j}\right),
$$

the full modeled transport equation for $a_{i j}$ is written as

$$
\begin{aligned}
\frac{D a_{i j}}{D t}=-\alpha_{1} \frac{\epsilon}{k} a_{i j}+\alpha_{2} \bar{S}_{i j} & +\alpha_{3}\left(a_{i l} \bar{S}_{l j}+\bar{S}_{i l} a_{l j}-\frac{2}{3} a_{n l} \bar{S}_{n l} \delta_{i j}\right) \\
& -\alpha_{4}\left(a_{i l} \bar{W}_{l j}-\bar{W}_{i l} a_{l j}\right)+\frac{1}{k}\left[D_{i j}-\left(a_{i j}+\frac{2}{3} \delta_{i j}\right) D\right],
\end{aligned}
$$

where $P_{i j}$ has been exactly expressed in terms of $a_{i j}, \bar{S}_{i j}$, and $\bar{W}_{i j}$. The tensor $\bar{W}_{i j}$ is the anti-symmetric part of the velocity gradient tensor, defined as

$$
\bar{W}_{i j} \equiv \frac{1}{2}\left(\frac{\partial \bar{u}_{i}}{\partial x_{j}}-\frac{\partial \bar{u}_{j}}{\partial x_{i}}\right)
$$

and the $\alpha_{i}$ in (9) are given as

$$
\alpha_{1}=\left(1+C_{1}^{0}\right) \frac{P}{\epsilon}-1+C_{1}^{1}, \quad \alpha_{2}=C_{2}-\frac{4}{3}, \quad \alpha_{3}=C_{3}-1, \quad \alpha_{4}=C_{4}-1 .
$$

The turbulence kinetic energy production $P$ appearing in (11) is given by

$$
P \equiv-\overline{u_{i}^{\prime} u_{j}^{\prime}} \bar{S}_{i j}
$$

or in terms of $a_{i j}$ as

$$
P=-k a_{i j} \bar{S}_{i j}
$$

The $C_{i}$ coefficients in (11) are given by the particular model for the pressure-strain rate correlation in (8), and are taken as

$$
C_{1}^{0}=0, \quad C_{1}^{1}=1.5, \quad C_{2}=0.8, \quad C_{3}=0.875, \quad C_{4}=0.655,
$$

in the Launder, Reece \& Rodi (LRR) model, ${ }^{2}$ and as

$$
C_{1}^{0}=0.9, \quad C_{1}^{1}=1.7, \quad C_{2}=0.8-0.65\left(I I_{a}\right)^{1 / 2}, \quad C_{3}=0.625, \quad C_{4}=0.2,
$$

in the Speziale, Sarkar \& Gatski (SSG) model, ${ }^{3}$ where $I I_{a} \equiv a_{n l} a_{l n}$ is the second invariant of the anisotropy tensor.

Reynolds stress transport (RST) models involve the solution of the full set of six coupled partial differential equations for $a_{i j}$ represented by (9). Closure of (9) is typically achieved through solution of two additional equations for the turbulence variables $k$ and $\epsilon$, as in standard two-equation turbulence models. However, it has already been noted in Section I that such RST approaches are often accompanied by numerical issues, and simpler algebraic approaches for representing the anisotropy, such as the equilibrium Boussinesq closure in (6), are more commonly used in simulations of practical applications. 


\section{II.B. Derivation of Nonequilibrium Closure and Effective Strain Rate}

Here we seek to retain the principal anisotropy dynamics contained in (9) within a relatively simple representation for $a_{i j}$ such as that used in (6). From the full modeled transport equation for $a_{i j}$ in (9), we propose a simplified quasi-linear dynamical equation for the anisotropy evolution as ${ }^{1}$

$$
\frac{D a_{i j}}{D t}=-\frac{1}{\Lambda_{m}} a_{i j}+\alpha_{2} \bar{S}_{i j},
$$

where $\Lambda_{m}$ is a turbulence memory time scale defined as

$$
\Lambda_{m} \equiv \frac{1}{\alpha_{1}} \tau_{T}
$$

with $\tau_{T}$ the characteristic turbulence time scale whose formulation varies depending on the turbulence variables (either $k-\epsilon$ or $k-\omega$ ) employed in the closure framework. While (16) lacks many of the higherorder interactions appearing in the full anisotropy transport equation in (9), it still contains the primary nonequilibrium $\left(D a_{i j} / D t\right)$, relaxation $\left(-a_{i j} / \Lambda_{m}\right)$, and rapid strain $\left(\alpha_{2} \bar{S}_{i j}\right)$ dynamics governing the evolution of the anisotropy tensor. Note that the equilibrium anisotropy closure in (6) is obtained from this equation by neglecting the nonequilibrium dynamics and setting $D a_{i j} / D t=0$.

Letting $\Lambda_{m}$ be a constant with respect to the timescale over which (16) must be integrated, the solution for $a_{i j}$ in (16) is a convolution integral of the form

$$
a_{i j}(t)=\int_{-\infty}^{t} \alpha_{2} \bar{S}_{i j}(\tau) e^{-(t-\tau) / \Lambda_{m}(t)} D \tau .
$$

By noting that ${ }^{8,9}$

$$
\frac{\nu_{T}}{k} \equiv-\frac{\alpha_{2}}{2} \Lambda_{m}
$$

the convolution in (18) can be equivalently written as

$$
a_{i j}=-2 \frac{\nu_{T}}{k} \widetilde{S}_{i j}
$$

where $\widetilde{S}_{i j}(t)$ is the effective strain rate tensor defined as

$$
\widetilde{S}_{i j}(t)=\int_{-\infty}^{t} \bar{S}_{i j}(\tau) \frac{e^{-(t-\tau) / \Lambda_{m}(t)}}{\Lambda_{m}(t)} D \tau .
$$

The relation in (20) between the anisotropy tensor $a_{i j}$ and the effective strain rate tensor thus gives the general nonequilibrium anisotropy closure hypothesis which replaces the equilibrium anisotropy closure in (6). Comparison with (6) shows that (20) is still an eddy viscosity formulation, and that it differs from the classical Boussinesq equilibrium hypothesis only in that the anisotropy tensor is proportional to the effective strain rate tensor $\widetilde{S}_{i j}$ in (21), rather than to the local instantaneous mean strain rate tensor $\bar{S}_{i j}$.

It has been shown previously ${ }^{1}$ that the nonequilibrium model given by (20) and (21) provides dramatically improved results over conventional closure approaches based on (6) in a range of nonequilibrium homogeneous test cases, including impulsively-sheared turbulence, periodically-sheared turbulence, turbulence that is strained, relaxed, and destrained, and turbulence interacting with a normal shock wave. In these homogeneous tests the nonequilibrium closure has been implemented in the standard $k$ - $\epsilon$ framework ${ }^{10}$ where the eddy viscosity is given as

$$
\nu_{T}=C_{\mu} \frac{k^{2}}{\epsilon}
$$

with $k$ and $\epsilon$ obtained through solution of the standard modeled transport equations valid for homogeneous flows, namely

$$
\begin{gathered}
\frac{d k}{d t}=P-\epsilon, \\
\frac{d \epsilon}{d t}=\left(C_{\epsilon 1} P-C_{\epsilon 2} \epsilon\right) \frac{\epsilon}{k},
\end{gathered}
$$


where $P$ is given in (13). In Ref. [1], the model constants were given the standard values ${ }^{10}$

$$
C_{\mu}=0.09, \quad C_{\epsilon 1}=1.44, \quad C_{\epsilon 2}=1.92,
$$

and despite the dependence of $\alpha_{1}$ on $P / \epsilon$ in (11), it was found that the constant value

$$
\frac{1}{\alpha_{1}} \equiv C_{\Lambda}=0.26
$$

yielded good agreement with validation data for all nonequilibrium test cases. The resulting memory time scale in the $k-\epsilon$ formulation is then

$$
\Lambda_{m}=C_{\Lambda} \frac{k}{\epsilon}
$$

\section{II.C. Time-Local Formulation of Nonequilibrium Model}

In order to evaluate the effective strain integral in (21) within a computational framework where only local instantaneous variables are available, the integral must be written in an equivalent time-local form that allows $\widetilde{S}_{i j}$ to be readily evaluated in such implementations. The Lagrangian strain rate history term $\bar{S}_{i j}(\tau)$ in the convolution integral in (21) can be expanded about the current time $t$, resulting in

$$
\widetilde{S}_{i j}(t)=\int_{-\infty}^{t} \frac{e^{-(t-\tau) / \Lambda_{m}}}{\Lambda_{m}}\left[\bar{S}_{i j}(t)-\left.\frac{D \bar{S}_{i j}}{D t}\right|_{t}(t-\tau)+\left.\frac{1}{2} \frac{D^{2} \bar{S}_{i j}}{D t^{2}}\right|_{t}(t-\tau)^{2}+\cdots\right] D \tau .
$$

Since the derivatives of $\bar{S}_{i j}$ do not depend on $\tau$, the integrals appearing in (28) are readily solved, which yields

$$
\widetilde{S}_{i j}(t)=\bar{S}_{i j}(t)+\left.\sum_{n=1}^{\infty}\left(-\Lambda_{m}\right)^{n} \frac{D^{n} \bar{S}_{i j}}{D t^{n}}\right|_{t} .
$$

The form of the effective strain rate tensor in (29) is equivalent to the convolution integral in (21), but allows a time-local evaluation of $\widetilde{S}_{i j}(t)$. With the nonequilibrium anisotropy hypothesis in (20), the time-local form in (29) allows straightforward implementation of the present nonequilibrium model in a computational framework where only local instantaneous variables are available.

From (20) and (29), the time-local nonequilibrium anisotropy closure suitable for implementation in traditional computational RANS modeling frameworks is thus written in general form as

$$
a_{i j}=-2 \frac{\nu_{T}}{k} \bar{S}_{i j}-2 \frac{\nu_{T}}{k} \sum_{n=1}^{\infty}\left(-\Lambda_{m}\right)^{n} \frac{D^{n} \bar{S}_{i j}}{D t^{n}}
$$

and the corresponding full Reynolds stress closure is

$$
\overline{u_{i}^{\prime} u_{j}^{\prime}}=\frac{2}{3} k \delta_{i j}-2 \nu_{T} \bar{S}_{i j}-2 \nu_{T} \sum_{n=1}^{\infty}\left(-\Lambda_{m}\right)^{n} \frac{D^{n} \bar{S}_{i j}}{D t^{n}} .
$$

Implementation of this closure model in two-equation RANS model frameworks is straightforward, and (30) or (31) directly replace the Boussinesq hypothesis wherever it appears. While in previous work ${ }^{1}$ the eddy viscosity $\nu_{T}$ in (30) and (31) has been determined by the underlying $k-\epsilon$ or $k$ - $\omega$ model, in Section III a realizable eddy viscosity modification based on a rigorous analysis of the modeled anisotropy transport equation in (9) will be presented. In the following we will consider periodically-sheared homogeneous turbulence in order to evaluate the convergence of truncated forms of the time-local expression for the anisotropy in (30) to the full convolution integral form represented by (20) and (21).

\section{II.D. Time-Local Formulation in Periodically-Sheared Homogeneous Turbulence}

To avoid issues of numerical stability and computational complexity as an increasing number of terms are retained in the expression for the anisotropy in (30), it is of interest to consider how well a truncated form of (30) reproduces the results of the full convolution anisotropy closure in (20) and (21). This analysis can be 
carried out through consideration of initially isotropic periodically-sheared homogeneous turbulence, where the applied mean strain rate is defined ${ }^{7}$ as

$$
\bar{S}_{12}(t)=\bar{S}_{21}(t)=\left\{\begin{array}{ll}
0 & \text { for } t<0 \\
\left(S_{\max } / 2\right) \sin (\omega t) & \text { for } t \geq 0
\end{array} .\right.
$$

This test case provides insight into the accuracy of the time-local formulation of the new closure over a range of nonequilibrium flow conditions (characterized by the magnitude of the shearing frequency $\omega / S_{\max }$ ), as well as the convergence of a reduced form of the time-local anisotropy in (30) to the full convolution anisotropy in (20) and (21).

Using the full convolution form of the anisotropy in (20) and (21), the shear anisotropy $a_{12}$ for the periodic shearing in (32) is given ${ }^{1}$ as

$$
a_{12}(t)=-\frac{\nu_{T}}{k} S_{\max }\left[\frac{1}{1+\left(\omega \Lambda_{m}\right)^{2}}\right]\left[\sin (\omega t)-\omega \Lambda_{m} \cos (\omega t)+\omega \Lambda_{m} e^{-t / \Lambda_{m}}\right] .
$$

It is clear from this expression that the nonequilibrium anisotropy has a frequency-dependent amplitude, phase shift, and offset, in contrast to the shear anisotropy predicted by the equilibrium Boussinesq hypothesis in (6), namely

$$
a_{12}(t)=-\frac{\nu_{T}}{k} S_{\max } \sin (\omega t)
$$

Considering the time-local formulation of the anisotropy from (30), the first five terms in the series $(n=5)$ yield

$$
a_{12}(t)=-\frac{\nu_{T}}{k} S_{\max }\left[1-\left(\omega \Lambda_{m}\right)^{2}+\left(\omega \Lambda_{m}\right)^{4}+\cdots\right]\left[\sin (\omega t)-\omega \Lambda_{m} \cos (\omega t)\right] .
$$

The sequence $\left[1-\left(\omega \Lambda_{m}\right)^{2}+\left(\omega \Lambda_{m}\right)^{4}+\cdots\right]$ in (35) is the Taylor expansion about the parameter $\left(\omega \Lambda_{m}\right)^{2}$ of the frequency-dependent amplitude term $\left[1+\left(\omega \Lambda_{m}\right)^{2}\right]^{-1}$ in (33). The frequency-dependent phase shift is primarily accounted for by the $\left[\omega \Lambda_{m} \cos (\omega t)\right]$ term in (35), and so inclusion of higher-order terms in (35) serves only to yield better amplitude agreement with the full convolution form in (33).

As shown previously, ${ }^{1}$ phase results obtained using the full anisotropy closure in (33), where the closure has been implemented within the $k-\epsilon$ model framework outlined in (22)-(27), agree much more closely with the DNS results from Yu and Girimaji ${ }^{7}$ than results obtained using the classical Boussinesq hypothesis, as shown in Figure 1. The phase difference $\phi$ is defined as the separation between the zero crossing points of the applied mean shear $\bar{S}_{12}$ in (32) and the shear anisotropy $a_{12}$. Considering the $n=1$ truncated form of the time-local nonequilibrium anisotropy from (35), namely

$$
a_{12}(t)=-\frac{\nu_{T}}{k} S_{\max }\left[\sin (\omega t)-\omega \Lambda_{m} \cos (\omega t)\right],
$$

Figure 1 shows that (36) reproduces the phase difference predicted by the full nonequilibrium model in (33) over a wide range of shearing frequencies. While there is some disagreement between the phase predicted by (33) and (36) at intermediate frequencies, the truncated form for $a_{12}$ in (36) is still in much better agreement with the DNS results ${ }^{7}$ than the Boussinesq expression for $a_{12}$ in (34) over all shearing frequencies.

Moreover, Figure 2 shows that the anisotropy evolution for shearing frequencies $\omega / S_{\max }=0.125$, $\omega / S_{\max }=0.25$, and $\omega / S_{\max }=0.5$ predicted by the $n=1$ truncation in (36) is also in good agreement with the full convolution integral form for $a_{12}$ in (33). The agreement between the truncated and full forms is particularly good for the low frequency cases in Figs. $2 a, b$, although there is a noticeable amplitude disagreement in Fig. $2 c$ for the $\omega / S_{\max }=0.5$ case. This disagreement is due to the neglect of the higher-order amplitude terms in obtaining (36) from (35). This indicates - not surprisingly given the expansion in (35) that as the degree of nonequilibrium in a turbulent flow increases (which is characterized by the parameter $\omega \Lambda_{m}$ in periodically sheared turbulence), a greater number of terms in the time-local anisotropy expansion in (30) must be retained to yield physically-accurate results. Nevertheless, while better amplitude agreement between the full and truncated expressions for $a_{12}$ in (33) and (35) is obtained by retaining higher-order terms in the expansion for $\widetilde{S}_{12}$, Figures 1 and 2 do indicate that even the $n=1$ truncated nonequilibrium closure in (36) is sufficient to obtain significantly higher-fidelity results for periodically-sheared turbulence than the classical Boussinesq hypothesis in (6). This suggests that a truncated $n=1$ form of the anisotropy closure in (30), where numerical difficulties are not expected to be prohibitive, is sufficient to give good results for practical nonequilibrium turbulent flow problems. 


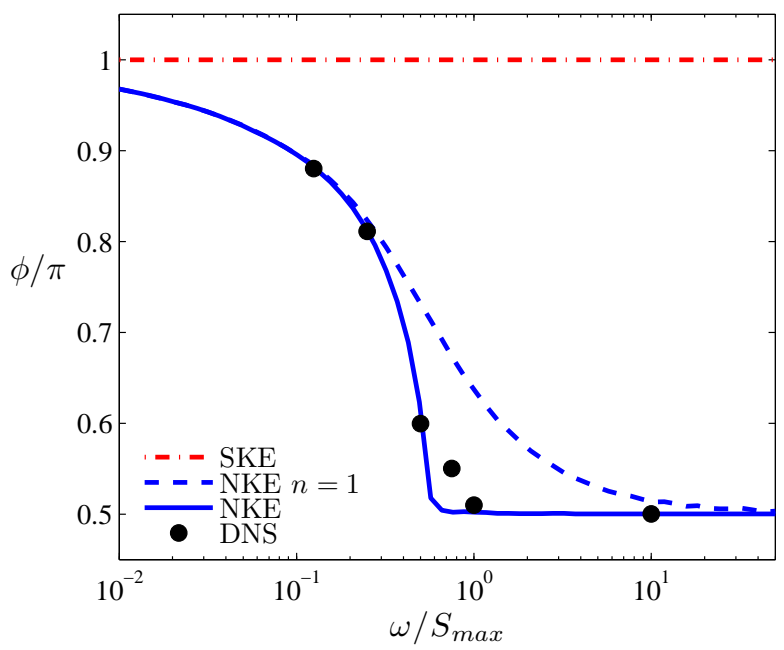

Figure 1. Phase difference between anisotropy $a_{12}$ and imposed mean shear $\bar{S}_{12}$ in periodically-sheared turbulence, comparing results obtained from $n=1$ truncated nonequilibrium closure in (36) (NKE $n=1$ ) with standard $k-\epsilon$ model in (34) (SKE), full nonequilibrium closure in (33) (NKE), and DNS results from Yu and Girimaji. ${ }^{7}$
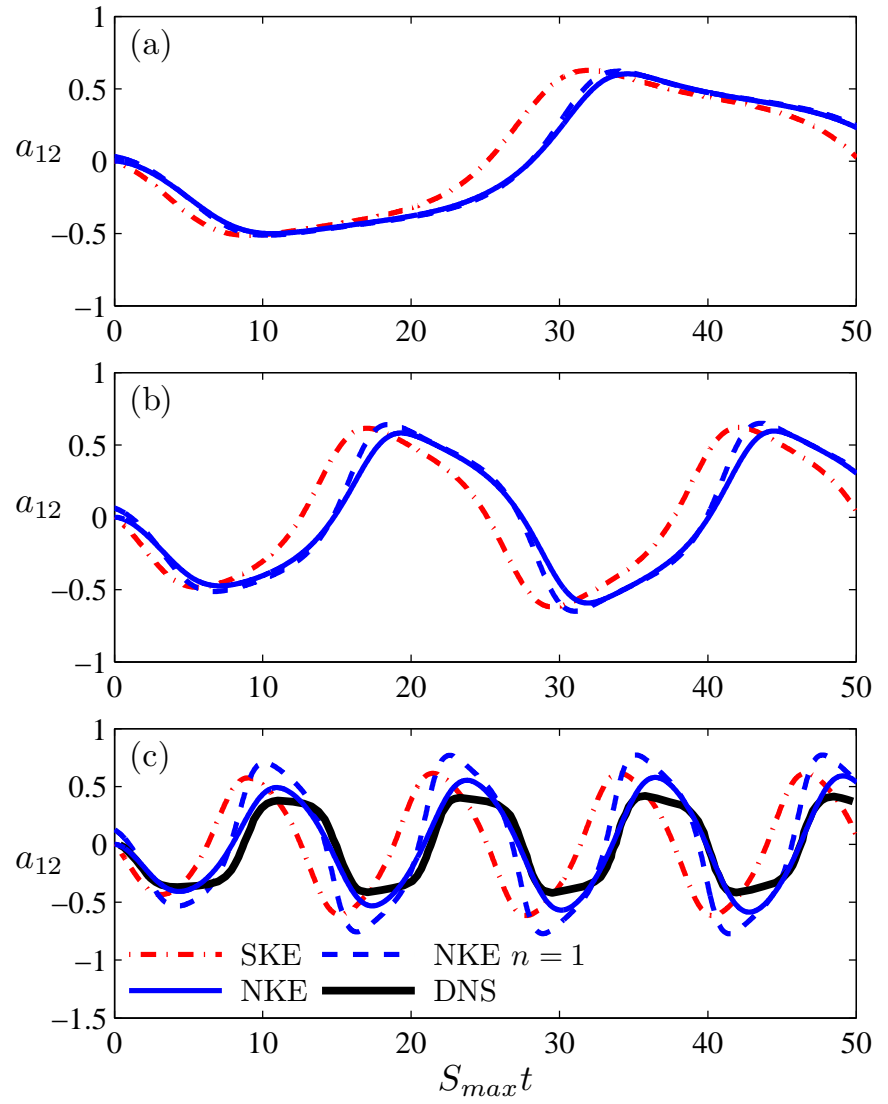

Figure 2. Temporal evolution of anisotropy $a_{12}$ for shearing frequencies (a) $\omega / S_{\max }=0.125$, (b) $\omega / S_{\max }=0.25$, and (c) $\omega / S_{\max }=0.5$, showing results from $n=1$ truncated nonequilibrium closure in (36) (NKE $\left.n=1\right)$, standard $k-\epsilon$ model in (34) (SKE), and full nonequilibrium closure in (33) (NKE). DNS results from Yu and Girimaji ${ }^{7}$ are shown in (c). 


\section{Realizable Eddy Viscosity for Nonequilibrium Closure}

It is well known that the assumption of a constant eddy viscosity coefficient $C_{\mu}$, as used for example in the standard $k-\epsilon$ framework in (22)-(25), is generally inaccurate in flows where the production-to-dissipation ratio $P / \epsilon$ becomes large. This can be seen explicitly by considering the definition of the eddy viscosity obtained from (17) and (19) for a $k$ - $\epsilon$ framework, namely

$$
\nu_{T}=-\frac{\alpha_{2}}{2 \alpha_{1}} \frac{k^{2}}{\epsilon} .
$$

It is clear from (11) with the coefficients $C_{i}$ given by the LRR model in (14) that $\alpha_{2}$ is a constant with value $\alpha_{2}=-8 / 15$, while $\alpha_{1}$ depends on $P / \epsilon$. By extension, (37) indicates that the eddy viscosity also explicitly depends on $P / \epsilon$. We can account for this dependence by noting that $P / \epsilon$ is given from (11) as

$$
\frac{P}{\epsilon}=\frac{\alpha_{1}}{1+C_{1}^{0}}+\frac{1-C_{1}^{1}}{1+C_{1}^{0}}
$$

and from the definition of $P$ in (13) as

$$
\frac{P}{\epsilon}=-\frac{k}{\epsilon} a_{i j} \bar{S}_{i j}=-\frac{\alpha_{2}}{\alpha_{1}}\left(\frac{k}{\epsilon}\right)^{2} \widetilde{S}_{i j} \bar{S}_{i j} .
$$

Defining the variable $\widetilde{C}_{\mu}$ as

$$
\widetilde{C}_{\mu}=-\frac{\alpha_{2}}{2 \alpha_{1}}
$$

we obtain a quadratic equation for $\widetilde{C}_{\mu}$ from (38) and (39), namely

$$
\widetilde{C}_{\mu}^{2}-\frac{1}{2 \eta^{2}}\left(\frac{1-C_{1}^{1}}{1+C_{1}^{0}}\right) \widetilde{C}_{\mu}+\frac{\alpha_{2}}{4 \eta^{2}\left(1+C_{1}^{0}\right)}=0,
$$

which has the solution

$$
\widetilde{C}_{\mu}=\left\{\begin{array}{ll}
\frac{\left(1-C_{1}^{1}\right)}{4 \eta^{2}\left(1+C_{1}^{0}\right)}\left[1-\left(1-4 \alpha_{2} \eta^{2} \frac{\left(1+C_{1}^{0}\right)}{\left(1-C_{1}^{1}\right)^{2}}\right)^{1 / 2}\right] & \text { for } \eta \neq 0 \\
\alpha_{2} / 2\left(1-C_{1}^{1}\right) & \text { for } \eta=0
\end{array},\right.
$$

where

$$
\eta \equiv \tau_{T} \sqrt{\widetilde{S}_{i j} \bar{S}_{i j}}
$$

Note that $k / \epsilon$ has been replaced with $\tau_{T}$ in (43) in order to obtain greater generality. The parameter $\eta$ contains nonequilibrium information in the form of the effective strain rate tensor $\widetilde{S}_{i j}$, in contrast to previous approaches for limiting the eddy viscosity that depend only on $\bar{S}_{i j}$. The resulting eddy viscosity coefficient $\widetilde{C}_{\mu}$ from (42) is limited to be no greater than the standard value $C_{\mu}^{*}=0.09$, namely

$$
C_{\mu}=\min \left[\widetilde{C}_{\mu}, C_{\mu}^{*}\right] .
$$

Figure 3 shows the dependence of $C_{\mu}$ from (42)-(44) on the parameter $\eta$, where the LRR model in (14) has been used to obtain values for $\alpha_{2}$ and the $C_{i}$ appearing in (42). It is clear from Figure 3 that at $\eta \approx 3.7$ the value of $C_{\mu}$ begins to decrease with increasing $\eta$. This is strongly reminiscent of realizable representations for $C_{\mu}$ such as the Bradshaw hypothesis, where $C_{\mu}$ is reduced for large values of $S k / \epsilon$ as

$$
C_{\mu}=\left\{\begin{array}{ll}
0.09 & \text { for } \quad(S k / \epsilon) \leq 3.4 \\
0.31(S k / \epsilon)^{-1} & \text { for } \quad(S k / \epsilon)>3.4
\end{array},\right.
$$

where $S \equiv\left[2 \bar{S}_{i j} \bar{S}_{i j}\right]^{1 / 2}$. Figure 3 shows that the current nonlinear form for $C_{\mu}$ in (42)-(44) is in relatively good agreement with the Bradshaw hypothesis in (45), where $C_{\mu}$ from (45) is plotted with respect to $S k / \epsilon$ instead of $\eta$. It should be noted however that $\widetilde{C}_{\mu}$ in (42) has been rigorously derived from the 


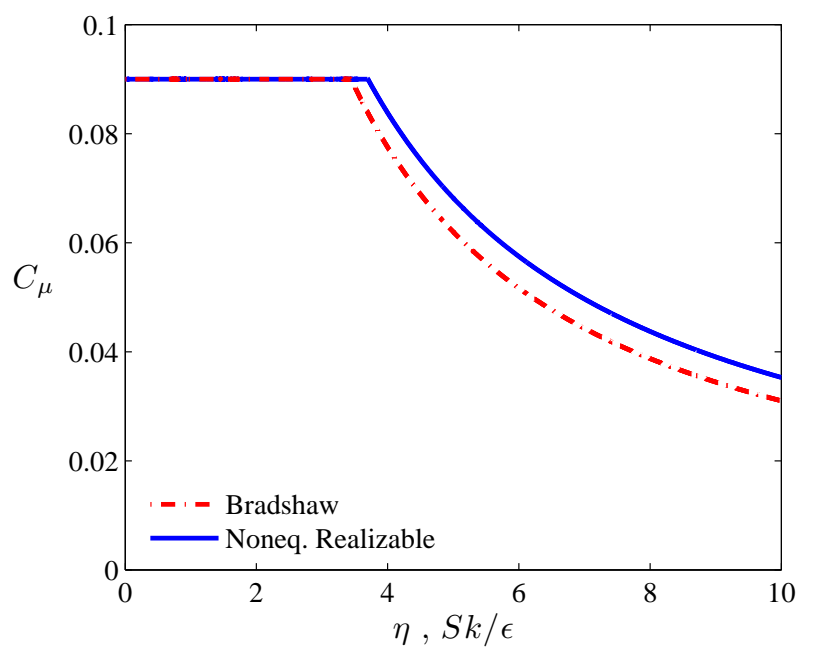

Figure 3. Realizable eddy viscosity coefficient $C_{\mu}$ as a function of $\eta$ or $S k / \epsilon$, as predicted by the realizable nonequilibrium model in (42)-(44) and the realizable Bradshaw hypothesis in (45).

modeled anisotropy transport equation in (9), and additionally accounts for nonequilibrium effects through the dependence of $\eta$ on $\widetilde{S}_{i j}$ as in (43). In the $k$ - $\epsilon$ framework the eddy viscosity is then given by

$$
\nu_{T}=C_{\mu} \frac{k^{2}}{\epsilon}
$$

while in the $k-\omega$ framework it is given by

$$
\nu_{T}=\frac{C_{\mu}}{C_{\mu}^{*}} \frac{k}{\omega}
$$

where in both cases $C_{\mu}$ is given by (42)-(44).

\section{III.A. Realizable Eddy Viscosity in Impulsively-Sheared Homogeneous Turbulence}

The accuracy of $C_{\mu}$ given by (42)-(44) is readily evaluated for impulsively-sheared homogeneous turbulence, where the applied mean shear is

$$
\bar{S}_{12}=\bar{S}_{21}=\left\{\begin{array}{ll}
0 & \text { for } t<0 \\
S / 2 & \text { for } t \geq 0
\end{array} .\right.
$$

While the equilibrium Boussinesq hypothesis in (6) predicts that the shear anisotropy is

$$
a_{12}=-\frac{\nu_{T}}{k} S
$$

the nonequilibrium closure based on the full convolution effective strain rate integral in (21) yields

$$
a_{12}=-\frac{\nu_{T}}{k} S\left[1-e^{-t / \Lambda_{m}}\right]
$$

It has already been shown ${ }^{1}$ that the nonequilibrium anisotropy in (50) provides better agreement with the LES validation results of Bardina et al. ${ }^{11}$ than the equilibrium anisotropy in (49) for the initial shear $S k_{0} / \epsilon_{0}=3.4$, where the eddy viscosity is represented in both (49) and (50) using the standard $k$ - $\epsilon$ framework as

$$
\nu_{T}=C_{\mu}^{*} \frac{k^{2}}{\epsilon}
$$

Now using the realizable eddy viscosity coefficient $C_{\mu}$ from (42)-(44) in place of $C_{\mu}^{*}$ in (51) for the nonequilibrium anisotropy in (50), we obtain the results for the kinetic energy evolution shown in Figure 4 . All models 

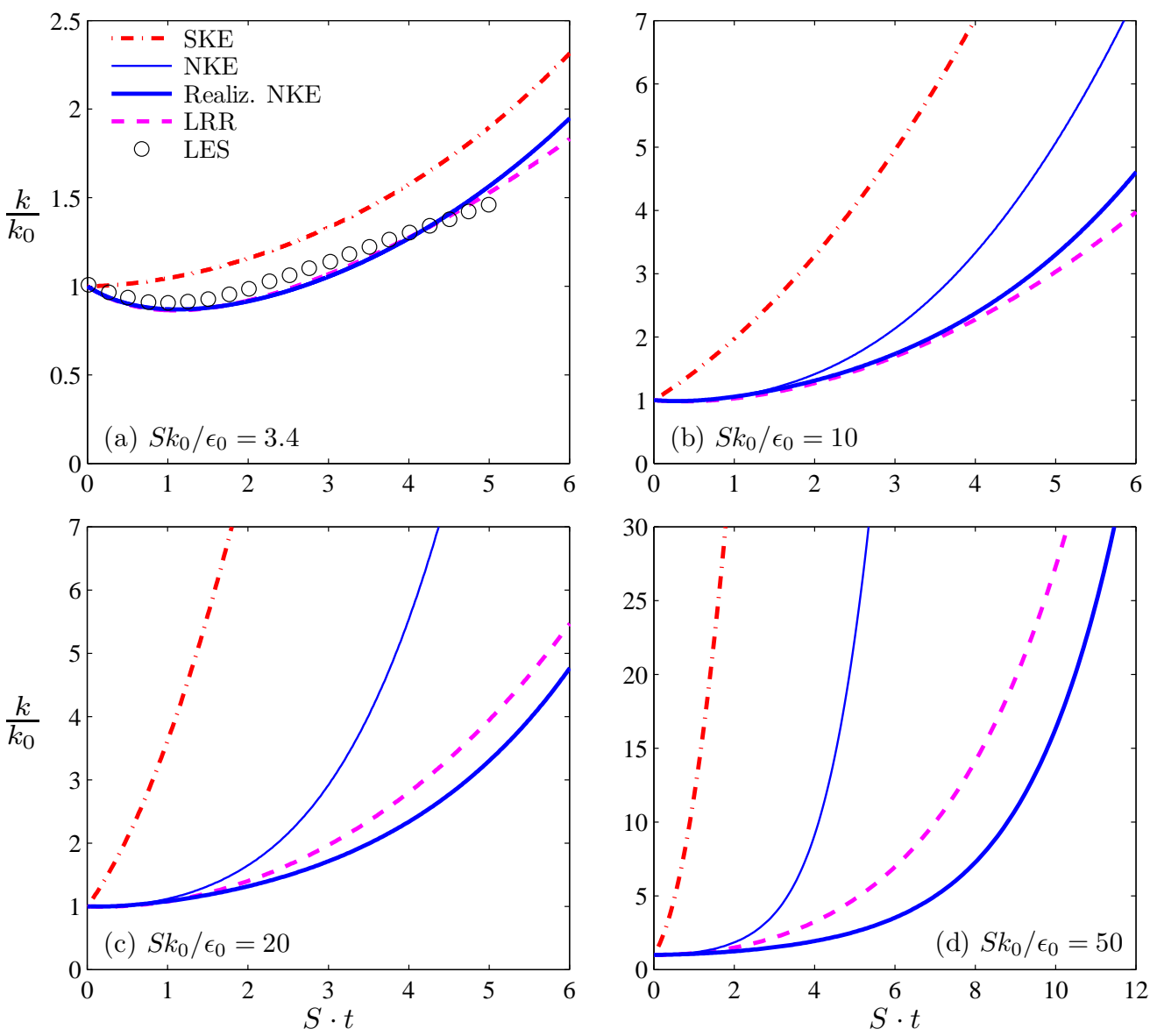

Figure 4. Kinetic energy evolution for initially-isotropic impulsively sheared turbulence for initial shearing frequencies (a) $S k_{0} / \epsilon_{0}=3.4$, (b) $S k_{0} / \epsilon_{0}=10$, (c) $S k_{0} / \epsilon_{0}=20$, and (d) $S k_{0} / \epsilon_{0}=50$. Results from the standard $k$ - $\epsilon$ model (SKE), the nonequilibrium $k-\epsilon$ model (NKE), and the nonequilibrium $k-\epsilon$ model with realizable $C_{\mu}$ given by (42)-(44) (Realizable NKE) are compared with results from the LRR RST model in (9) and (14). LES validation data from Bardina et al. ${ }^{11}$ are shown in (a).

have been implemented using the homogeneous $k$ - $\epsilon$ model framework outlined in (22)-(25). In Figure 4, results from the standard model in (49) with (51), nonequilibrium model in (50) with (51), and nonequilibrium model in (50) with realizable $C_{\mu}$ given by (42)-(44) are presented for initial shearing frequencies $S k_{0} / \epsilon_{0}=3.4$, $S k_{0} / \epsilon_{0}=10, S k_{0} / \epsilon_{0}=20$, and $S k_{0} / \epsilon_{0}=50$. In the sense that the realizable nonequilibrium closure approach presented herein is intended as an approximate solution to the full modeled anisotropy transport equation in (9), we generally require the realizable nonequilibrium closure results to be in good agreement with the underlying RST model, which in this case is the LRR model in (9) and (14). Subsequently, results from the LRR RST model are also shown in Figure 4 for comparison.

It is clear from Fig. $4 a$ that both forms of nonequilibrium closure provide better agreement with the LES validation results than the standard model in (49), particularly for small times. For the larger initial shear cases in Figs. $4 b-d$, both the standard and nonequilibrium anisotropy models substantially overpredict the kinetic energy magnitude when compared to the LRR model results. By contrast, the realizable nonequilibrium closure is in much better agreement with the LRR results and begins to show substantial departures from the LRR results only for the very large initial shear in Fig. $4 d$.

\section{Computational Formulation of the Nonequilibrium Anisotropy Closure}

The computational formulation of the nonequilibrium anisotropy closure with rigorously derived realizable eddy viscosity is given as

$$
\overline{u_{i}^{\prime} u_{j}^{\prime}}=\frac{2}{3} k \delta_{i j}+k a_{i j}
$$




$$
\begin{aligned}
& a_{i j}=-2 \frac{\nu_{T}}{k} \widetilde{S}_{i j} \\
& \widetilde{S}_{i j}=\bar{S}_{i j}+\left.\sum_{n=1}^{\infty}\left(-\Lambda_{m}\right)^{n} \frac{D^{n} \bar{S}_{i j}}{D t^{n}}\right|_{t} \\
& \Lambda_{m}=C_{\Lambda} \tau_{T} \\
& \widetilde{C}_{\mu}=\left\{\begin{array}{lll}
\frac{\left(1-C_{1}^{1}\right)}{4 \eta^{2}\left(1+C_{1}^{0}\right)}\left[1-\left(1-4 \alpha_{2} \eta^{2} \frac{\left(1+C_{1}^{0}\right)}{\left(1-C_{1}^{1}\right)^{2}}\right)^{1 / 2}\right] & \text { for } & \eta \neq 0 \\
\alpha_{2} / 2\left(1-C_{1}^{1}\right) & \text { for } & \eta=0
\end{array}\right. \\
& \eta=\tau_{T} \sqrt{\widetilde{S}_{i j} \bar{S}_{i j}}, \\
& C_{\mu}=\min \left[\widetilde{C}_{\mu}, C_{\mu}^{*}\right] .
\end{aligned}
$$

The constants in (52)-(58) are taken in the following from the LRR model ${ }^{2}$ in (14) as

$$
C_{\mu}^{*}=0.09, \quad C_{\Lambda}=0.26, \quad C_{1}^{0}=0, \quad C_{1}^{1}=1.5, \quad \alpha_{2}=-\frac{8}{15},
$$

although other values such as those in (15) for the SSG model ${ }^{3}$ are also possible.

The closure formulation in (52)-(59) can be used in any two-equation turbulence modeling framework that provides a suitable expression for the eddy viscosity. In the widely-used standard $k-\epsilon$ and $k$ - $\omega$ model frameworks, the eddy viscosity $\nu_{T}$ and turbulence time scale $\tau_{T}$ are given as

$$
\nu_{T}=C_{\mu} \frac{k^{2}}{\epsilon}, \quad \tau_{T}=\frac{k}{\epsilon},
$$

for $k-\epsilon$ implementations and

$$
\nu_{T}=\frac{C_{\mu}}{C_{\mu}^{*}} \frac{k}{\omega}, \quad \tau_{T}=\frac{1}{C_{\mu}^{*} \omega},
$$

for $k$ - $\omega$ implementations.

\section{Computational Results Using Nonequilibrium Closure}

In the following, preliminary computational results obtained using the new nonequilibrium closure are presented for turbulent flow over a flat-plate, where results can be compared with the Spalding curve fit, ${ }^{12}$ and the interaction of an oblique shock wave impinging on a turbulent boundary layer. While the nonequilibrium anisotropy closure developed herein is formulated as a replacement for the equilibrium closure in (6), it can be implemented in essentially any two-equation turbulence model that employs that the Boussinesq hypothesis in (6). For initial testing, the present nonequilibrium closure will be implemented and evaluated within the framework of the Wilcox $k$ - $\omega$ two-equation turbulence model. ${ }^{6}$ This model is available in CFL3D and other codes for solving (1)-(4), and allows full integration to viscous walls. In the following, the standard $k-\omega$ model formulation with the nonequilibrium anisotropy closure used in preliminary testing will first be outlined, and then results for the two inhomogeneous test cases will be presented.

\section{V.A. Standard $k-\omega$ Model Framework}

The standard $k-\omega$ model of Wilcox ${ }^{6}$ contains essentially the same physics as standard $k-\epsilon$ model approaches, but is formulated in such a way as to make the near wall damping functions unnecessary. In the standard formulation, the eddy viscosity is given by the expression

$$
\nu_{T}=\frac{k}{\omega},
$$

where $\omega$ is a "turbulence frequency" that is proportional to $\epsilon / k$. The corresponding transport equations for $k$ and $\omega$ are then

$$
\begin{aligned}
& \frac{D k}{D t}=P-\beta^{\prime} k \omega+\frac{1}{\rho} \frac{\partial}{\partial x_{j}}\left[\left(\mu+\frac{\mu_{T}}{\sigma_{k}}\right) \frac{\partial k}{\partial x_{j}}\right], \\
& \frac{D \omega}{D t}=P_{\omega}-\beta \omega^{2}+\frac{1}{\rho} \frac{\partial}{\partial x_{j}}\left[\left(\mu+\frac{\mu_{T}}{\sigma_{\omega}}\right) \frac{\partial \omega}{\partial x_{j}}\right],
\end{aligned}
$$


where $P$ is defined as in (13), and $P_{\omega}=2 \gamma \bar{S}_{i j} \bar{S}_{j i}$. Standard values of the model constants are ${ }^{6}$

$$
\beta^{\prime}=0.09, \quad \beta=0.075, \quad \kappa=0.41, \quad \sigma_{k}=2.0, \quad \sigma_{\omega}=2.0, \quad \gamma=\frac{\beta}{\beta^{\prime}}-\frac{\kappa^{2}}{\sigma_{\omega} \sqrt{\beta^{\prime}}} .
$$

For the purposes of initial testing, the nonequilibrium closure is implemented within this framework by replacing the eddy viscosity in (62) with the nonequilibrium eddy viscosity in (61). The resulting kinetic energy production term $P$ is then

$$
P=2 \frac{C_{\mu}}{C_{\mu}^{*}} \frac{k}{\omega} \widetilde{S}_{i j} \bar{S}_{i j}
$$

and the $\omega$ production term $P_{\omega}$ is

$$
P_{\omega}=2 \frac{C_{\mu}}{C_{\mu}^{*}} \gamma \widetilde{S}_{i j} \bar{S}_{i j}
$$

where $C_{\mu}$ is given by (56)-(58). The effective strain rate in (54) is truncated at the $n=1$ term, yielding

$$
\widetilde{S}_{i j}=\bar{S}_{i j}-\Lambda_{m} \frac{D \bar{S}_{i j}}{D t}
$$

where for the $k-\omega$ model $\Lambda_{m}$ is given as

$$
\Lambda_{m}=\frac{C_{\Lambda}}{C_{\mu}^{*}} \frac{1}{\omega}
$$

with $C_{\Lambda}$ and $C_{\mu}^{*}$ defined as in (59). It was shown in Section II.D for periodically-sheared homogeneous turbulence that an $n=1$ truncation of the effective strain rate such as that in (68) was sufficient to give good modeled results for a range of shearing frequencies. In order to maintain computational stability using the truncated form of the effective strain rate, the quantity $\widetilde{S}_{i j} \bar{S}_{i j}$ in (67) and elsewhere is limited to be within $50 \%$ of $\bar{S}_{i j} \bar{S}_{i j}$. This restriction can be relaxed as additional terms are retained in the expansion for the effective strain rate tensor.

In the following, results for the nonequilibrium $k$ - $\omega$ model are compared with standard $k-\omega$ model results for inhomogeneous flat-plate and shock-boundary layer test cases. Implementation and testing of the nonequilibrium closure have been carried out in CFL3D, ${ }^{5}$ and calculation of the Lagrangian derivative of the mean strain rate tensor, namely $D \bar{S}_{i j} / D t$, is the most computationally intensive aspect of the new closure framework. Nevertheless, the overall computational complexity of the new closure approach is still substantially lower than full RST models.

\section{V.B. Flat-plate Boundary Layer}

Figure 5 shows the streamwise velocity in wall coordinates obtained using the nonequilibrium $k$ - $\omega$ model for incompressible $M=0.2$ boundary layer flow over a flat-plate. Nonequilibrium model results are compared with those obtained using the standard $k-\omega$ model.

The nonequilibrium $k-\omega$ results in Figure 5 show an exact correspondence with the underlying standard $k-\omega$ model, as is expected since $D \bar{S}_{i j} / D t=0$ along the flat-plate. Both the standard and nonequilibrium $k-\omega$ model results are in good agreement with the Spalding curve fit, ${ }^{12}$ as expected.

In a sense, the flat-plate boundary layer is a consistency check for the new model approach, since boundary layer and channel flows are essentially equilibrium flows where mean flow Lagrangian derivatives of flow properties are small, resulting in $\bar{S}_{i j}=\tilde{S}_{i j}$.

\section{V.C. Shock Interaction with Turbulent Boundary Layer}

From a turbulence modeling perspective, the interaction of a shock wave with a turbulent boundary layer remains relatively poorly understood. ${ }^{13,14}$ Studies of the interaction have traditionally focused on geometrically simple compression ramp and impinging oblique shock flows, but in recent years there has been increased interest in shock-boundary layer flow control for more sophisticated defense and aerospace applications. This has, in turn, led to a renewed effort within the scientific and engineering communities to develop improved turbulence models for simulation of the interaction, in part through more advanced direct numerical simulation (DNS) and experimental studies of basic shock-boundary layer flows. ${ }^{15,16}$ Impinging shock flows are particularly advantageous for the study of fundamental shock-boundary layer physics since 


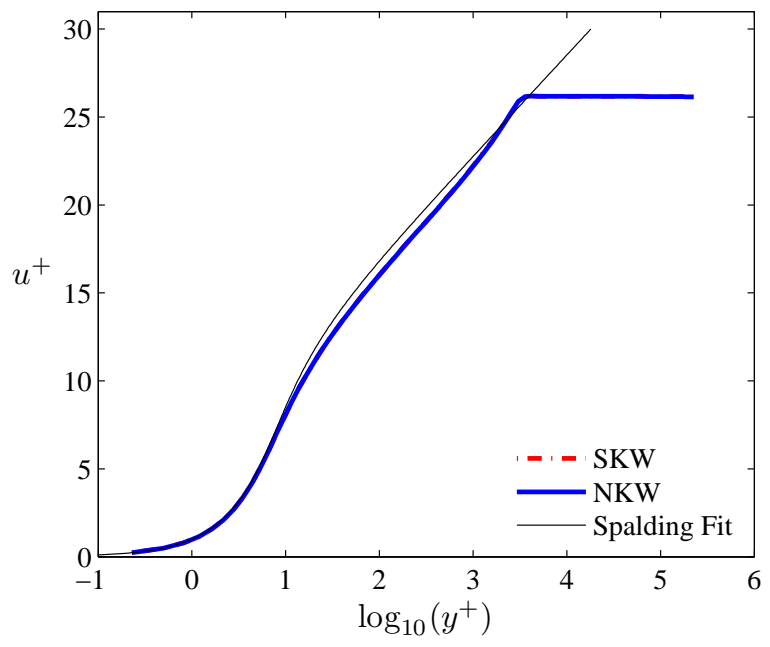

Figure 5. Mean streamwise velocity for turbulent flow over a flat-plate. The new nonequilibrium $k$ - $\omega$ results are compared with results from the standard $k-\omega$ model, ${ }^{6}$ as well as the Spalding curve fit. ${ }^{12}$

the interaction dynamics can be studied in the absence of curvature effects (which are present in compression ramp flows, and are a separate problem).

Currently the most poorly understood, and by extension poorly modeled, feature of impinging shock flows is the separation bubble that forms when a strong shock interacts with the turbulent boundary layer. Several researchers ${ }^{4,17,18}$ have described the failure of both linear eddy viscosity models and more advanced nonlinear eddy viscosity models in simulating impinging shock flows, and in particular the following three shortcomings have been noted:

(i) the location and extent of the separated flow region are poorly predicted computationally,

(ii) the turbulence kinetic energy both within and downstream of the interaction region is poorly predicted,

(iii) the location of the reflected shock emanating from the interaction region for an impinging oblique shock flow is often incorrect.

The third point is related to the first two, and if the simulated separation region and turbulence kinetic energy can be made more accurate then the location of the reflected shock will also improve.

In the following we apply the new nonequilibrium closure to the simulation of an $M_{1}=2.9$ oblique shock impinging on a turbulent boundary layer, where the flow deflection angle is $\theta=13^{\circ}$. This results in a relatively strong shock wave with an angle of $\beta=30.96^{\circ}$. A schematic of the simulation domain and boundary conditions is shown in Figure 6 , and flow parameters used to initialize the simulation are listed in Table 1. Only the region immediately surrounding the shock wave impingement point was calculated in the simulation. The interaction region was taken to be two-dimensional for the computations, and $x=0$ in the streamwise direction corresponds to the inviscid shock impingement point.

Figures 8-9 show results for the turbulence kinetic energy $k$, the Reynolds shear stress $\overline{u^{\prime} v^{\prime}}$, and the mean streamwise velocity $U$ obtained using the standard and nonequilibrium $k$ - $\omega$ models for the shock-turbulent boundary layer test case outlined in Table 1. All three Figures show that the interaction region predicted by the nonequilibrium $k-\omega$ model is much smaller than that predicted by the standard $k-\omega$ model. Figure 8 shows that the overall turbulence kinetic energy magnitude is greater for the nonequilibrium model than for the standard model, particularly downstream of the shock impingement point. Both the standard and nonequilibrium models predict a thickening of the boundary layer after the shock-boundary layer interaction, although the nonequilibrium model predicts a smaller post-interaction thickness compared to the standard model, as shown in Figure 9.

While there are substantial differences between the standard and nonequilibrium model results in Figures 8-9, comparison with high-quality experimental or computational validation data is required to fully assess the accuracy of both approaches. The current shock-boundary layer case is similar to the impinging shock 


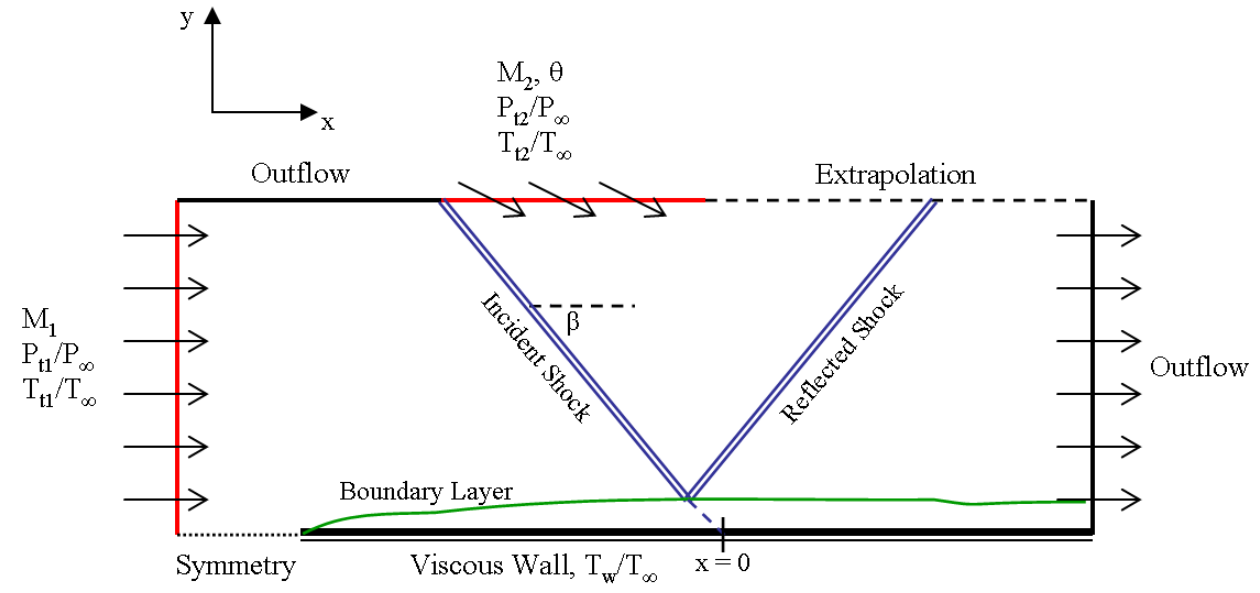

Figure 6. Schematic of the computational domain showing all boundary conditions and flow properties. The incoming pre-shock flow parameters at the left boundary are denoted by the subscript 1 and the post-shock flow entering the top of the computational domain at angle $\theta$ is denoted by 2 . The subscript ' $t$ ' denotes a stagnation variable, while the subscript ' $\infty$ ' denotes a freestream variable.

\begin{tabular}{lc|lc}
\hline \hline Parameter & Value & Parameter & Value \\
\hline$M_{1}$ & 2.9 & $M_{2}$ & 2.28 \\
$\theta\left(^{\circ}\right)$ & 13 & $P_{t 2} / P_{\infty}$ & 29.46 \\
$\beta\left(^{\circ}\right)$ & 30.96 & $T_{t 2} / T_{\infty}$ & 2.68 \\
$\operatorname{Re} / L_{\infty}\left(\times 10^{-6}\right)$ & 57 & $T_{t \infty}(K)$ & 291 \\
$P_{t 1} / P_{\infty}$ & 31.59 & $T_{\infty}(K)$ & 108.50 \\
$T_{t 1} / T_{\infty}$ & 2.68 & $T_{w} / T_{\infty}$ & 2.50 \\
\hline \hline
\end{tabular}

Table 1. Table of parameters used to initialize the SBLI computations. The incoming pre-shock flow properties are denoted by the subscript 1 while the post-shock flow properties are denoted by 2 . The subscript ' $t$ ' denotes a stagnation variable, while the subscript ' $\infty$ ' denotes a freestream variable. The post-shock flow deflection angle is $\theta$, and the shock angle is $\beta$, both measured in negative degrees with respect to the $x$ axis. The boundary layer width $\delta_{0} / L_{\infty}$ at the beginning of the interaction region is taken from the validation data and the wall temperature ratio $T_{w} / T_{\infty}$ is determined using an adiabatic wall with a recovery factor of $r_{f}=0.89$.

experiments of Reda \& Murphy ${ }^{19}$ and Modarress \& Johnson, ${ }^{20}$ for which only limited validation data are available. There is now a substantial need in the engineering community for high-quality validation data of strong oblique shock waves impinging on turbulent boundary layers. This would allow a more complete evaluation of the physical-accuracy of the new nonequilibrium closure approach. A full three-dimensional simulation using the nonequilibrium model - instead of the two-dimensional simulation presented herein is also required in order to assess the new model under more realistic flow conditions.

\section{Conclusions}

The full computational formulation of a new nonequilibrium anisotropy closure has been presented. The new closure accounts for strain history effects through an effective strain rate tensor, and also includes a realizable eddy viscosity that is rigorously derived from the governing anisotropy transport equation. The time-local representation and realizable eddy viscosity in the new nonequilibrium closure have been evaluated using fundamental tests of periodically and impulsively sheared homogeneous turbulence.

An initial form of the full nonequilibrium computational approach has been implemented in CFL3D within the standard $k-\omega$ model framework. As expected, the nonequilibrium $k-\omega$ model yields identical results to the standard $k-\omega$ model for boundary layer flow over a flat-plate, due to the fact that mean flow Lagrangian derivatives of $\bar{S}_{i j}$ are small. Results for an oblique shock wave impinging on a turbulent boundary layer, where the initial Mach number is $M_{1}=2.9$ and the flow deflection angle is $\theta=13^{\circ}$, indicate that the nonequilibrium model predicts increased kinetic energy within the interaction region, a smaller interaction 

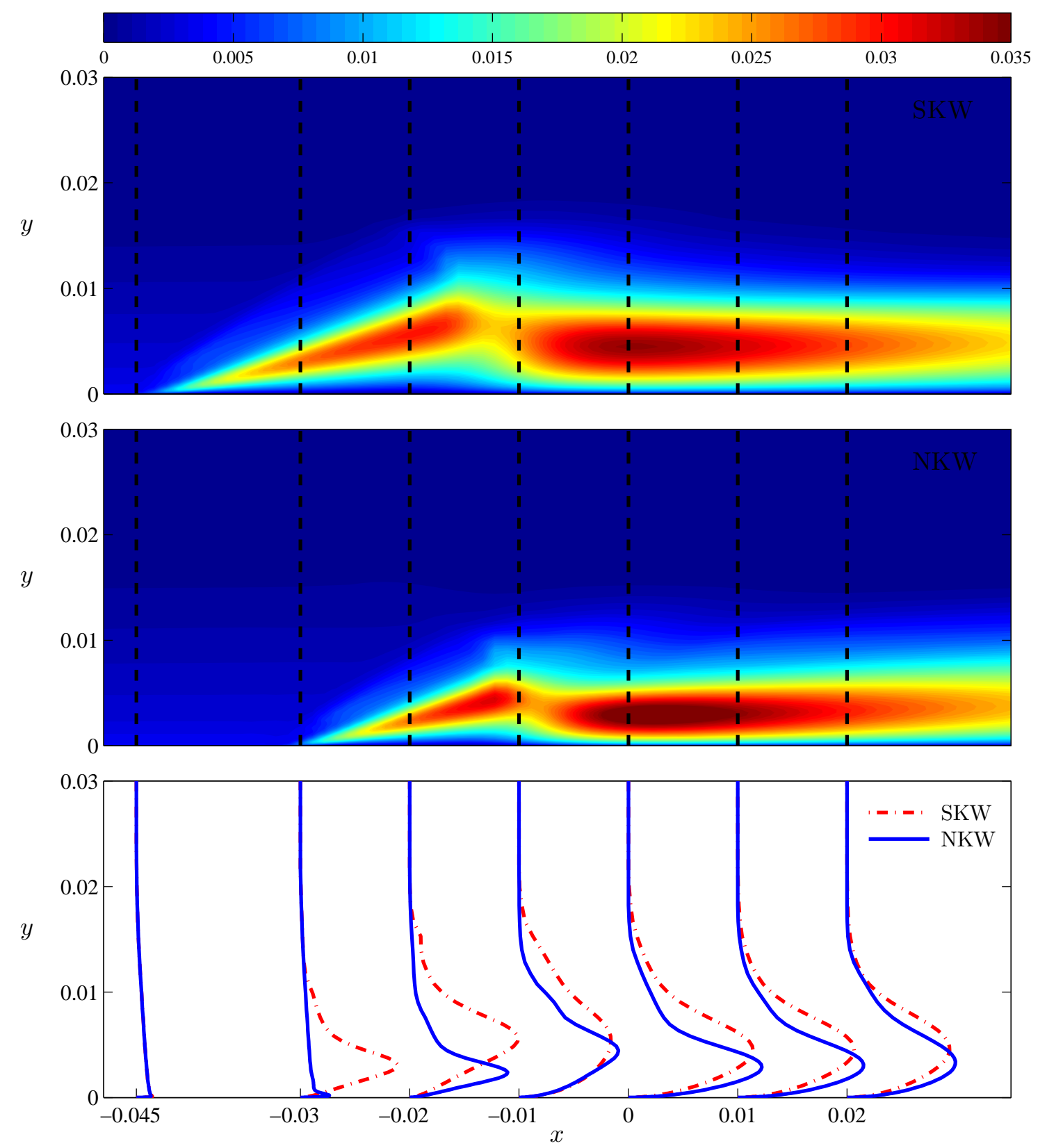

Figure 7. Profiles of the turbulence kinetic energy $k / U_{\infty}^{2}$ at various locations for the $M=2.9, \theta=13^{\circ}$ shock-boundary layer test case using standard and nonequilibrium $k-\omega$ models. The top panel shows kinetic energy contours obtained using the standard model, and the middle panel shows contours using the nonequilibrium model. 

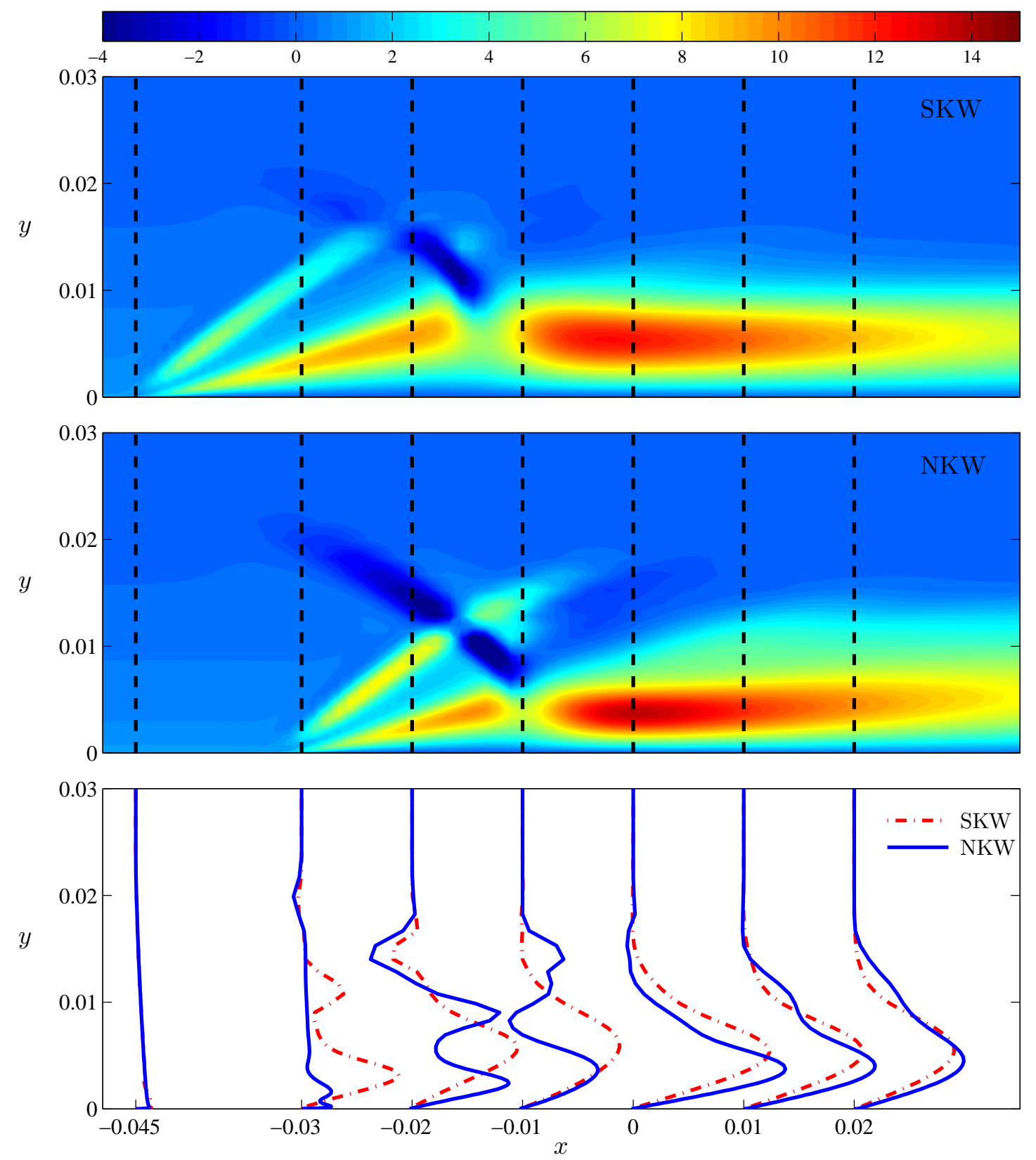

Figure 8. Profiles of the Reynolds shear stress $-\overline{u^{\prime} v^{\prime}} / U_{\infty}^{2} \times 10^{3}$ at various locations for the $M=2.9$, $\theta=13^{\circ}$ shockboundary layer test case using standard and nonequilibrium $k-\omega$ models. 

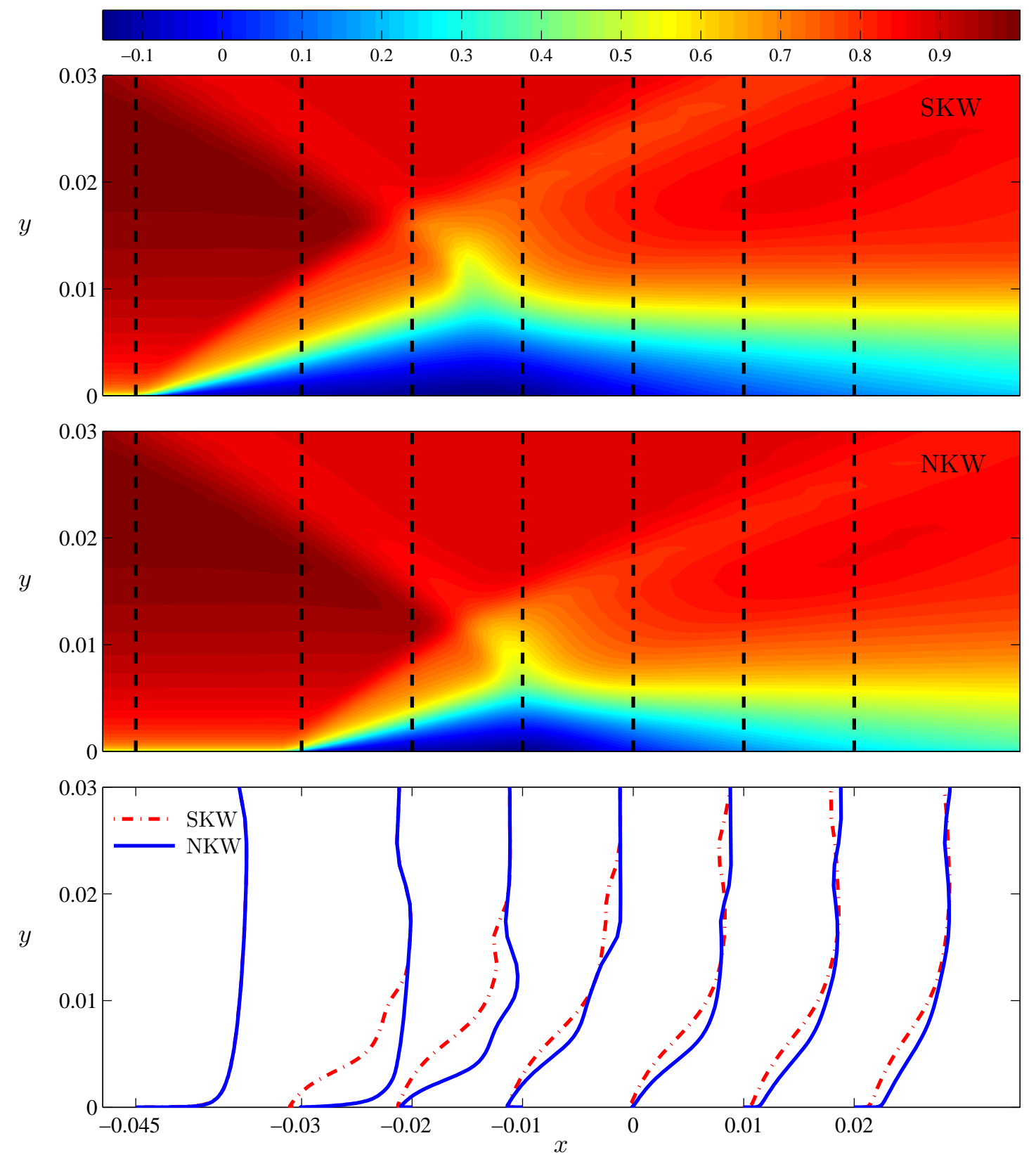

Figure 9. Profiles of the mean streamwise velocity $U / U_{\infty}$ at various locations for the $M=2.9, \theta=13^{\circ}$ shock-boundary layer test case using standard and nonequilibrium $k-\omega$ models. 
region, and a thinner post-interaction boundary layer.

\section{Acknowledgements}

This work was supported, in part, by the Air Force Research Laboratory (AFRL) through the MichiganAFRL Collaborative Center for Aeronautical Sciences (MACCAS) under Award No. FA8650-06-2-3625, and by the National Aeronautics \& Space Administration (NASA) Marshall and Glenn Research Centers and the Department of Defense (DoD) under the NASA Constellation University Institutes Project (CUIP) under Grant No. NCC3-989.

\section{References}

\footnotetext{
${ }^{1}$ Hamlington, P. E. and Dahm, W. J. A., "Reynolds stress closure for nonequilibrium effects in turbulent flows." Phys. Fluids, Vol. 20, 115101, 2008.

${ }^{2}$ Launder, B. E., Reece, G., and Rodi, W., "Progress in the development of a Reynolds stress turbulence closure." J. Fluid Mech., Vol. 68, 1975, pp. 537-566.

${ }^{3}$ Speziale, C. G., Sarkar, S., and Gatski, T. B., "Modeling the pressure strain correlation of turbulence: an invariant dynamical systems approach." J. Fluid Mech., Vol. 227, 1991, pp. 245-272.

${ }^{4}$ Rizzetta, D. P., "Evaluation of explicit algebraic Reynolds-stress models for separated supersonic flows." AIAA Journal, Vol. 36 (1), 1998, pp. 24-30.

${ }^{5}$ Rumsey, C., Biedron, R., and Thomas, J., "CFL3D: Its history and some recent applications." NASA TM, 1997, pp. 112861.

${ }^{6}$ Wilcox, D. C., Turbulence Modeling for CFD, DCW Industries, 2000.

${ }^{7} \mathrm{Yu}$, D. and Girimaji, S. S., "Direct numerical simulations of homogeneous turbulence subject to periodic shear." J. Fluid Mech., Vol. 566, 2006, pp. 117-151.

${ }^{8}$ Gatski, T. B. and Speziale, C. G., "On explicit algebraic stress models for complex turbulent flows." J. Fluid Mech., Vol. 254, 1993, pp. 59-78.

${ }^{9}$ Yoshizawa, A., Nisizima, S., Shimomura, Y., Kobayashi, H., Matsuo, Y., Abe, H., and Fujiwara, H., "A new methodology for Reynolds-averaged modeling based on the amalgamation of heuristic-modeling and turbulence-theory methods." Phys. Fluids, Vol. 18, 2006, pp. 035109.

${ }^{10}$ Launder, B. E. and Spalding, D. B., "The numerical computation of turbulent flows." Comp. Meth. App. Mech. Eng., Vol. 3, 1974, pp. 269-289.

${ }^{11}$ Bardina, J., Ferziger, J. H., and Reynolds, W. C., "Improved turbulence models based on large-eddy simulation of homogeneous, incompressible turbulent flows." Rep. No. TF-19, Vol. Stanford University, 1983.

${ }^{12}$ White, F. M., Viscous Fluid Flow, McGraw-Hill, 1991.

${ }^{13}$ Dolling, D. S., "50 years of shock wave/boundary layer interaction - what next?" AIAA Paper, Vol. 2000-2596, 2000.

${ }^{14}$ Dolling, D. S., "Unsteadiness of shock-induced turbulent separated flows - Some key questions." AIAA Paper, Vol. 2001$2708,2001$.

${ }^{15}$ Pirozzoli, S. and Grasso, F., "Direct numerical simulation of impinging shock wave/turbulent boundary layer interaction at $\mathrm{M}=2.25 . "$ Phys. Fluids, Vol. 18, 2006.

${ }^{16}$ Humble, R. A., Scarano, F., and van Oudheusden, B. W., "Particle image velocimetry measurements of a shock wave/turbulent boundary layer interaction." Exp. Fluids, Vol. 43, 2007, pp. 173-183.

${ }^{17}$ Liou, W. W., Huang, G., and Shih, T. H., "Turbulence model assessment for shock wave/turbulent boundary-layer interaction in transonic and supersonic flows." Comp. Fluids, Vol. 29, 2000, pp. 275-299.

${ }^{18}$ Gerolymos, G. A., Sauret, E., and Vallet, I., "Oblique-shock-wave/boundary-layer interaction using near-wall Reynolds stress models." AIAA Journal, Vol. 42 (6), 2004, pp. 1089-1100.

${ }^{19}$ Reda, D. C. and Murphy, J. D., "Shock-Wave/Turbulent-Boundary Layer Interactions in Rectangular Channels." AIAA Journal, Vol. 11 (2), 1973, pp. 139-140.

${ }^{20}$ Modarress, D. and Johnson, D. A., "Investigation of turbulent boundary-layer separation using laser velocimetry." $A I A A$ Journal, Vol. 17 (7), 1979, pp. 747-752.
} 